\title{
Ayudas públicas como determinante de la cooperación en investigación y desarrollo
}

\author{
Guisado-González, Manue** \\ Guisado-Tato, Manuel** \\ Ferro-Soto, Carlos***
}

\begin{abstract}
Resumen
El objetivo de este artículo es analizar los determinantes de la cooperación en investigación y desarrollo (I+D) de las empresas innovadoras del sector manufacturero español, prestando especial atención a las ayudas públicas a la innovación, una variable poco frecuente en esta clase de estudios académicos. Los datos utilizados en el estudio proceden del Panel de Innovación Tecnológica del año 2010 (PITEC, 2010). El análisis empírico se llevó a cabo mediante la realización de una regresión logística binaria de naturaleza bietápica, con la finalidad de eliminar el potencial sesgo de selección que pudiera estar presente. Los resultados indican que las empresas que exhiben una mayor probabilidad de cooperar son aquellas que reciben alguna clase de ayuda pública a la innovación, realizan mayores gastos internos en investigación y desarrollo (I+D), pertenecen a un grupo de empresas, tienen un mayor tamaño y utilizan métodos de protección legal para evitar que otras empresas se apropien de los resultados de sus investigaciones. La principal conclusión del estudio es que la concesión de ayudas públicas a la innovación, bajo la condicionalidad de establecer acuerdos de cooperación debe practicar una cierta clase de discriminación entre sectores de alta y baja intensidad tecnológica.
\end{abstract}

Palabras clave: Innovación, ayudas públicas, cooperación en Investigación y Desarrollo.

Recibido: 14-02-13. Aceptado: 20-07-13

* Doctor en administración y dirección de empresas. Profesor adscrito al departamento de organización de empresas y marketing, Universidad de Vigo, España. e-mail: mguisado@uvigo.es

** Doctor en administración y dirección de empresas Profesor adscrito al departamento de organización de empresas y marketing, Universidad de Vigo, España.

*** Doctor en administración y dirección de empresas Profesor titular adscrito al departamento de organización de empresas y marketing, Universidad de Vigo, España. 


\title{
Public Assistance as a Determinant for Cooperation in Research and Development
}

\begin{abstract}
The objective of this article is to analyze the determinants for cooperation in research and development $(R+D)$ at innovative enterprises in the Spanish manufacturing sector, paying special attention to public assistance for innovation, an infrequent variable in this type of academic study. Data utilized in the study come from the Panel for Technological Innovation of 2010 (PITEC, 2010). The empirical analysis was carried out by performing a binary logistical regression of the bi-stage type, in order to eliminate the potential bias of selection that could exist. Results indicate that companies exhibiting a greater probability of cooperation are those that receive some type of public assistance for innovation, making greater internal expenditures in research and development $(R+D)$; they belong to a group of companies, have a larger size and utilize legal protection methods to avoid that other companies appropriate their research results. The main conclusion of this study is that the concession of public assistance for innovation, under the condition of establishing cooperation agreements, ought to practice a certain kind of discrimination between sectors of high and low technological intensity.
\end{abstract}

Keywords: Innovation, public assistance, cooperation in research and development.

\section{Introducción}

En la literatura económica suele ser frecuente señalar que los motivos que empujan a las empresas a cooperar en I+D son básicamente tres: aprovechar flujos de conocimiento e información relevantes (Belderbos et al., 2004), reducir costes y riesgos (Miotti y Sachwald, 2003) y la búsqueda de complementariedades (Hagedoorn, 1993). Además, suele señalarse que el aprovechamiento adecuado de los motivos primero y tercero requiere la posesión previa de una amplia y experimentada capacidad de absorción (Cohen y Levinthal, 1989,1990).

Al respecto, son numerosos los trabajos empíricos que han tenido en cuenta, en mayor o menor medida, los anteriores motivos a la hora de analizar los determinantes de los acuerdos de cooperación (Fritsch y Lukas, 2001; Tether, 2002;
Miotti y Sachwald, 2003; Belderbos et al, 2004; Bönte y Keilbach, 2005, Bayona et al., 2006; López, 2008).

En relación al aprovechamiento de los flujos de conocimiento e información (spillovers), cumple resaltar que la actividad de I+D es imperfectamente apropiable, lo que determina que los resultados de investigación y desarrollo de las empresas pueden ser total o parcialmente disfrutados por empresas competidoras que no han realizado esfuerzo inversor alguno (Arrow, 1959).

Esta característica de los bienes y servicios tecnológicos reduce los incentivos de las empresas a emprender actividades de I+D. Sin embargo, la literatura económica ha enfatizado que las actividades de I+D resultan estrictamente necesarias desde la perspectiva del bienestar social de las poblaciones, ya que son precisamente las sociedades más avan- 
zadas tecnológicamente las que disfrutan en general de mayor bienestar y calidad de vida. Por tanto, si las actividades de I+D fueran totalmente abandonadas al libre juego de las fuerzas de mercado es posible que la inversión en I+D que realizan las empresas sea inferior al nivel óptimo socialmente deseable. La existencia de spillovers que las empresas no pueden controlar constituye una de las principales razones que empuja a las empresas a cooperar y a las administraciones gubernamentales a incentivar y promover acuerdos de cooperación en I+D.

Consecuentemente, a partir del reconocimiento de que los acuerdos de cooperación en I+D contribuyen a una más eficaz y rápida difusión de la tecnología y de los flujos de conocimientos, una parte considerable de los fondos públicos nacionales y de la Unión Europea (UE) destinados a la promoción de la innovación están siendo dirigidos a estimular acuerdos de cooperación entre empresas y entre éstas y diferentes organismos públicos dedicados a la investigación en I+D (Acosta y Modrego, 2001; Abramovsky et al., 2004).

Aunque resultan escasos los estudios que han utilizado esta variable como determinante de la propensión a cooperar de las empresas, parece pertinente comprobar si los amplios fondos públicos destinados a este menester están consiguiendo el objetivo de estimular los acuerdos de cooperación. Al respecto, Falk (2004) señala que los estímulos fiscales a la investigación y al desarrollo tecnológico tienen la potencialidad de modificar la propensión a cooperar con otras empresas y organismos públicos de investigación.
El objetivo principal de este estudio es contribuir al análisis de los principales determinantes de los acuerdos de cooperación en $\mathrm{I}+\mathrm{D}$ en el contexto del sector manufacturero español, prestando especial atención sobre el particular a la intervención de las diferentes administraciones públicas implicadas en el fomento y apoyo de las actividades de innovación.

Las variables que se utilizan en el estudio (Cooperación, Apoyo público, Tamaño, Intensidad en I+D, Spillovers entrantes, Costos y riesgos, Complementariedades, Métodos de protección legal, Grupo) proceden de los microdatos del Panel de Innovación Tecnológica 2010 (PITEC, 2010). Esta base de datos está elaborada por el Instituto Nacional de Estadística de España (INE) siguiendo las directrices establecidas en el Manual de Oslo (OECD y Eurostat, 1997). Los microdatos de PITEC proceden de la Community Innovation Survey (CIS). La población a la cual se aplicó PITEC 2010 estuvo conformada por 12821 empresas españolas. De esta población, el sector manufacturero está conformado por 5221 empresas, mientras que las manufactureras innovadoras totalizan la cifra de 4311 empresas.

Cara a contrastar las hipótesis formuladas se utilizó la muestra de las 4311 empresas manufactureras innovadoras, y empleamos como técnica estadística la regresión logística binaria. La Cooperación es la variable dependiente, mientras que las demás variables son independientes. Ahora bien, dado que estamos restringiendo el análisis al universo de empresas manufactureras innovadoras, en la medida en que son esta clase de empresas las que despliegan de modo 
explícito diferentes estrategias de innovación, ello puede dar lugar a problemas de sesgo de selección.

Esta clase de sesgo puede tener lugar cuando la variable de selección empleada (en este caso, ser o no empresa innovadora) es observada en lugar de estimada, lo que provoca que en el modelo a estimar no se encuentren presentes los efectos de las empresas no innovadoras que pueden tener influencia sobre la decisión de cooperar en I+D. Para corregir este sesgo suele utilizarse el denominado método de corrección Heckman. Sin embargo, cuando la variable dependiente es binaria, y por tanto no continua, existen autores que recomiendan un método de corrección similar, aunque distinto (Greene, 1993; Hass y Hansen, 2005).

El método, propuesto por Manning et al. (1987), utiliza dos modelos y se materializa en dos etapas: en la primera etapa emplea el denominado modelo de selección, en el que la variable dependiente, también denominada variable de selección, es de naturaleza binaria y mide la probabilidad de que la empresa en cuestión sea innovadora. Por otro lado, las variables independientes del modelo de selección deben tener algún grado de explicación sobre la probabilidad de innovar. En esta primera etapa, se regresa la variable de selección sobre el conjunto de variables explicativas ${ }^{1}$, a fin de determinar para cada empresa su correspondiente probabilidad de innovar.

La regresión se practica sobre la totalidad de las empresas manufactureras, sean o no innovadoras (5221 empresas). En la segunda etapa se utiliza el denominado modelo de interés, formado por las variables definidas en el apéndice $A$ y por la probabilidad de innovar de cada empresa, calculada en la etapa anterior. En este caso, la regresión se practica sobre el conjunto de empresas innovadoras (4311 empresas). De este modo, los coeficientes obtenidos en esta segunda fase habrán sido corregidos de los correspondientes sesgos de selección que pudieran estar presentes.

1 En este estudio, se incluyen en el modelo de selección variables idénticas o similares a otros estudios que han analizado la probabilidad de ser una empresa innovadora (Cassiman y Veugelers, 2006); a tal efecto, incluimos como variables explicativas: Tamaño, Grupo, Intensidad en I+D y un total de diez variables que miden los obstáculos a la innovación que perciben las empresas (falta de fondos internos, falta de financiación externa, costes de innovación, falta de personal cualificado, falta de información sobre tecnología, falta de información sobre mercados, dificultad para encontrar socios para cooperar en innovación, mercado dominado por empresas establecidas, incertidumbre de la demanda de los bienes innovadores, actividad innovadora no necesaria por la existencia de innovaciones previas y actividad innovadora no necesaria por falta de demanda de innovaciones). Asimismo, como el sector manufacturero está integrado por muchos subsectores, es necesario controlar la influencia de los mismos sobre la probabilidad de innovar. Por ello, en la correspondiente ecuación de selección, también se incluyen como variables explicativas un conjunto de variables ficticias relacionadas con la pertenencia a cada uno de los correspondientes subsectores que integran el sector manufacturero. Estos subsectores están definidos al nivel de dos dígitos de la Nomenclatura de Actividades Económicas de la Comunidad Europea (NACE). 


\section{Ayudas públicas, innovación y acuerdos de cooperación en investigación y desarrollo}

La concesión de subsidios a la innovación bajo la obligación de establecer acuerdos de cooperación con otras empresas persigue la consecución de dos objetivos simultáneamente por parte de las administraciones públicas: asegurar un nivel razonable y mínimo de rentabilidad para que muchas empresas sigan generando innovaciones y tratar de asegurar que el conocimiento generado, aunque sea parcialmente, sea difundido.

En el ámbito de las ayudas públicas a la innovación, a través del fomento de acuerdos de cooperación entre empresas innovadoras, y entre éstas y diferentes organismos públicos centrados en la investigación, los estudios empíricos realizados no son muy numerosos.

Sobre el particular, Kleinknecht y Reijnen (1992) y Reger y Kuhlmann (1995) llegan a la conclusión de que las ayudas a la innovación han incrementado el número de acuerdos de cooperación que las empresas innovadoras afectadas han materializado, contribuyendo de este modo a la difusión de la tecnología, mientras que Gemünden et al. (1992) señalan que la participación de las empresas en los diferentes programas de ayuda pública a la innovación puede constituir una cabal explicación del incremento de los acuerdos de cooperación entre empresas. Georghiou y Roessner (2000) y Luukkonen (2000), han analizado la participación de empresas europeas en programas de ayudas públicas a la innovación en un contexto de cooperación, encontrando un efecto positivo sobre la capacidad de innovación de las empresas que han cooperado.

Asimismo, cumple destacar que diferentes estudios han analizado la efectividad de las ayudas públicas a la innovación utilizadas como herramienta de política pública que busca promover los acuerdos de cooperación entre empresas a fin de mejorar el bienestar de la correspondiente sociedad $^{2}$ (Yi y Shin, 2000).

Dado que los acuerdos de cooperación contribuyen a la difusión de la tecnología y, por tanto, a la generación de externalidades para la sociedad (Heijs, 2005), y que las autoridades públicas están interesadas en mejorar el bienestar de las sociedades bajo sus égidas, cabe esperar que los sistemas de ayudas públicas a la innovación, instrumentados a través de las correspondientes agencias públicas, tengan una influencia positiva

2 La promoción de los acuerdos de cooperación en I+D constituyen un elemento clave de las denominadas políticas de bienestar, en la medida en que la posesión de un tejido productivo de elevada intensidad tecnológica contribuye a la existencia de empresas competitivas, y por tanto generadoras de empleo, y a la fabricación de productos de mayor calidad y/o más baratos para los consumidores. Por ejemplo, el objetivo principal del $5^{\circ}$ Programa Marco de la Unión Europea perseguía de modo explícito la promoción de proyectos que contribuyeran a la mejora de la capacidad de innovación de las empresas y el bienestar de los ciudadanos. 
sobre el despliegue de actividades cooperativas en I+D por parte de las empresas innovadoras.

Por ello, y teniendo en cuenta que en España son tres las fuentes genéricas de financiación pública posibles (europea, estatal y autonómica), parece oportuno contrastar la hipótesis de que las ayudas públicas a la innovación influyen significativamente en la probabilidad de cooperar de las empresas, y tratar de analizar sobre el particular la contribución de cada una de las mencionadas agencias públicas.

Por otra parte, en la literatura sobre cooperación empresarial son muchos los trabajos empíricos que han relacionado la propensión a cooperar con la existencia de una capacidad previa de I+D, en la medida en que desde la Teoría de la capacidad de absorción se señala que para absorber eficazmente y con rapidez los conocimientos y tecnologías y habilidades que los socios puedan aportar es necesario poseer previamente una amplia capacidad de aprendizaje (Cohen y Levinthal, 1990). Por consiguiente, las empresas poseedoras de elevados niveles de capacidad de absorción serán más eficaces a la hora de crear y explotar las relaciones que establecen con otras empresas (Caloghirou et al., 2004).

La literatura sobre innovación señala que la capacidad de absorción depende fuertemente del actual nivel de conocimientos tecnológicos que se poseen (Kim, 2001), el cual a su vez deriva de los esfuerzos en I+D interna que previamente se hayan acometido (Stock et al., 2001; Schoenmakers and Duysters, 2006). Sobre el particular, se señala que una insufi- ciente capacidad de absorción dificulta la habilidad de las empresas para reconocer el potencial de las nuevas ideas que pudieran surgir en su interacción con otros socios cooperantes (Schoenmakers and Duysters, 2006; Tsai, 2009).

En general, trabajos empíricos como los de Arora y Gambardella (1994), Colombo y Garrone (1998) y Fritsch y Lukas (2001) han encontrado que las empresas que realizan investigación interna de un modo sistemático muestran una mayor propensión a materializar acuerdos de cooperación con otras empresas u organizaciones públicas de investigación. Asimismo, otros muchos estudios han encontrado evidencias de que la intensidad en I+D es una variable que influye en la probabilidad de contraer acuerdos de cooperación (Bayona et al., 2002; Tether, 2002; Miotti and Sachwald, 2003; Negassi, 2004; Sampson, 2007; Dachs et al, 2008; Faria et al., 2010). Por tanto, en consonancia con la argumentación precedente, en este trabajo se busca contrastar que existe una relación positiva y significativa entre la intensidad en I+D de las empresas y la probabilidad de establecer acuerdos de cooperación.

Por otra parte, cabe subrayar que la relación entre spillovers y acuerdos de cooperación en I+D ha sido ampliamente estudiada. Sobre el particular, el trabajo seminal de D'Aspremont y Jacquemin (1988) ha sido el primero en señalar que las empresas cooperan en I+D con el fin de internalizar los spillovers que se generan; es decir, a través de la instrumentación de acuerdos de cooperación en I+D las empresas buscan que las fugas no deseadas de información favorezcan 
principalmente a sus socios, y que las fugas que éstos generan les favorezcan a ellas.

Estos acuerdos de cooperación, además de internalizar los spillovers alivian la duplicación de esfuerzos e inversiones por parte de los socios cooperantes, ya que los mismos persiguen, aunque sea parcialmente, unos mismos objetivos. Por consiguiente, se espera que los spillovers entrantes tengan una influencia positiva sobre la probabilidad de cooperar en I+D (Cassiman y Veugelers, 2002; López, 2008).

En relación a los métodos legales y estratégicos de protección la literatura existente sugiere la existencia de impactos contradictorios (López, 2008). Por un lado, unas menores barreras de protección empujan a las empresas a cooperar, a fin de internalizar los correspondientes spillovers; por otro, una menor protección también facilita la parasitación sobre las inversiones en I+D que realizan otras empresas, cuestión que disminuye la propensión a cooperar, en la medida en que resulta factible la consecución de conocimiento tecnológico sin necesidad de cooperar con otras empresas. A priori, en este trabajo no se establece ninguna hipótesis sobre el impacto que los métodos de protección legal tienen sobre la probabilidad de cooperar.

Los acuerdos de cooperación permiten compartir los costes y los riesgos que toda actividad de I+D comporta (Hite y Hesterly, 2001), lo que facilita que las empresas implicadas inviertan menos recursos y asuman menos riesgos que si emprenden las actividades de I+D de un modo independiente (Das y Ten, 2000). Por consiguiente, cuantos mayores sean los costes de inversión en I+D y mayores sean los riesgos percibidos, mayor será la probabilidad de que las empresas establezcan acuerdos de cooperación en I+D.

Resulta evidente que las empresas buscan en la cooperación en I+D compartir información tecnológica con el objeto de reducir la incertidumbre que toda actividad de I+D conlleva. Pero para que la acción de compartir información sea fructífera, la información compartida debe ser complementaria, es decir, la información no debe ser totalmente redundante (Anbarci et al., 2002). Al respecto, existen evidencias empíricas de que la presencia de un cierto grado de complementariedad entre las empresas que cooperan en I+D incrementa la propensión de las mismas a establecer acuerdos de cooperación (Cowan y Jonard, 2008).

Ahora bien, para detectar extramuros la existencia de conocimiento complementario es necesario que la empresa posea internamente elevados conocimientos tecnológicos. Consecuentemente, suele asumirse que la disponibilidad de un amplio conocimiento tecnológico a nivel interno favorece la probabilidad de cooperación en I+D (López, 2008).

Las empresas pueden competir en los mercados como unidades independientes o formando parte de grupos empresariales. En general, la pertenencia a un grupo empresarial conlleva una serie de ventajas, tales como la probabilidad de beneficiarse de la difusión tecnológica entre las distintas unidades empresariales que conforman el grupo, la posibilidad de disfrutar de elevadas economías de alcance (Henderson y Cockburn, 1996), tener acceso a una mayor disponibilidad de recursos financieros, y un mayor apoyo y 
respaldo en los procesos de negociación en los que la empresa se encuentre involucrada.

Sobre el particular, cumple reseñar que autores como Tether (2002), Miotti y Sachwald (2003), Mohnen y Hoareau (2003), Belderbos et al. (2004) y Okamuro et al. (2011) encontraron que la pertenencia a un grupo empresarial incrementa las probabilidades de cooperar en I\&D con otras empresas u organismos públicos. En este trabajo se espera un similar comportamiento.

Finalmente, señalar que en este estudio se utiliza como variable control el tamaño de la empresa. También en este caso no se hace ninguna conjetura previa sobre su influencia sobre la probabilidad de cooperar en I+D, ya que la misma resulta contradictoria. Por un lado, cabe esperar que las grandes empresas dispongan de los recursos necesarios para encontrar el socio conveniente (Cohen and Levinthal, 1990) pero, por otro, cabe esperar que si disponen de amplios recursos no necesitarán cooperar para conseguir nuevos conocimientos (Cassiman and Veugelers, 2002).

\section{Empresas manufactureras: Impacto de las ayudas públicas sobre la cooperación en investigación y desarrollo}

Previo al análisis de los resultados, en necesario precisar las variables utilizadas en el estudio y su tratamiento en el contexto de la investigación:

- Cooperación: Cuando la empresa no realiza actividades de cooperación la variable toma el valor cero, y uno cuando coopera a nivel de I+D con otras empresas o instituciones.

- Apoyo público: Esta variable toma el valor cero si la empresa no participa en ningún programa de ayuda pública a la innovación durante el período de análisis; el valor 1 cuando participa únicamente en programas de las Comunidades Autónomas españolas; el valor 2 cuando participa únicamente en el programa estatal español; el valor 3 cuando participa únicamente en el programa europeo; y el valor 4 cuando participa simultáneamente en más de uno de los programas anteriores.

- Tamaño: Logaritmo de la cifra de negocios.

- Intensidad en I+D: Ratio entre gastos internos en I+D y cifra de negocios.

- Spillovers entrantes. Valor medio de las puntuaciones (1-4) otorgadas por las empresas a dos fuentes de información (Congresos, ferias y exposiciones; Revistas científicas y publicaciones técnicas) sobre proyectos de innovación (1, no empleada; 4, muy importante).

- Costes y riesgos: Valor medio de las puntuaciones (1-4) otorgadas por las empresas a cuatro ítems (Falta de fondos internos; Falta de financiación externa; Costes de innovación elevados; Demanda incierta de bienes innovadores) que evalúan obstáculos a la innovación de naturaleza económico-financiera (1, no relevante; 4, muy importante).

- Complementariedades: Se evalúan mediante la puntuación que otorga la empresa (1-4) a la falta de información sobre la tecnología como un obstáculo 
a la innovación (1, muy importante; 4, no relevante). Diversos autores (Cassiman y Veugelers, 2002; Belderbos et al., 2004; López, 2008) denominan a esta variable Complementariedades, ya que la búsqueda de complementariedades tecnológicas depende de la disponibilidad de información tecnológica a nivel interno.

- Métodos de protección legal: Suma de las puntuaciones (0 ó 1) otorgadas por las empresas a cuatro métodos de protección legal (Patentes; Registro de modelos de utilidad; Marcas de fábrica; Derechos de autor) (0, no empleado; 4, muy importante).

- Grupo: Cuando la empresa forma parte de un grupo de empresas esta variable toma el valor 1. Cuando la empresa es independiente, la variable toma el valor 0 .

Teniendo en cuenta cada una de las variables, se procede a la presentación, análisis y discusión de los resultados obtenidos en la investigación:

En la Tabla 1, se presentan los estadísticos descriptivos de las variables que forman parte de la ecuación de interés. De un total de 4311 empresas manufactureras innovadoras, 1421 empresas (33\%) tienen acuerdos de cooperación en I+D, mientras que 2890 empresas (67\%) no cooperan. Los valores medios de todas las variables son superiores para las empresas que cooperan en I+D frente a las que no cooperan, a excepción expresa de la variable complementariedades, cuyo valor medio es ligeramente superior para las empresas que no cooperan.

En general, las diferencias entre ambas clases de empresas son muy elevadas, principalmente en lo que concierne a las variables apoyo público, intensidad en I+D, métodos de protección legal y grupo. De acuerdo con estos estadísticos, resulta evidente que las empresas

Tabla 1

Estadísticos descriptivos: Modelo de interés

\begin{tabular}{|c|c|c|c|c|c|c|}
\hline & \multirow[t]{2}{*}{$\begin{array}{l}\text { Valor } \\
\text { mín. }\end{array}$} & \multirow[t]{2}{*}{$\begin{array}{l}\text { Valor } \\
\text { máx. }\end{array}$} & \multicolumn{2}{|c|}{$\begin{array}{c}\text { COOPERAN } \\
\text { (1421 empresas } 33 \%)\end{array}$} & \multicolumn{2}{|c|}{$\begin{array}{c}\text { NO COOPERAN } \\
(2890 \text { empresas }-67 \%)\end{array}$} \\
\hline & & & Media & Desv. típ. & Media & Desv. típ. \\
\hline Apoyo público & 0 & 4 & 1,5004 & 1,5038 & 0,4824 & 1,0391 \\
\hline Spillovers entrantes & 1 & 4 & 2,3543 & 0,8506 & 1,9194 & 0,8906 \\
\hline Intensidad en I+D & 0 & 3,52 & 0,0496 & 0,1784 & 0,0233 & 0,0882 \\
\hline Costes y riesgos & 1 & 4 & 2,9029 & 0,7395 & 2,8670 & 0,8493 \\
\hline Complementariedades & 1 & 4 & 2,6981 & 0,8081 & 2,7782 & 0,8600 \\
\hline $\begin{array}{l}\text { Métodos de } \\
\text { protección legal }\end{array}$ & 0 & 4 & 0,6122 & 0,8758 & 0,3626 & 0,7358 \\
\hline Grupo & 0 & 1 & 0,5600 & ,04960 & 0,3600 & 0,4810 \\
\hline Tamaño & 3,78 & 9,69 & 7,2091 & 0,8125 & 6,8480 & 0,7433 \\
\hline
\end{tabular}

Fuente: Elaboración propia a partir de la Base de Datos PITEC (2010). 
innovadoras del sector manufacturero español que cooperan reciben un mayor apoyo público que las que no cooperan, a la par que evidencian una mayor complejidad tecnológica.

En la Tabla 2, se exponen los resultados obtenidos de la regresión logística binaria practicada sobre la ecuación de interés, una vez eliminados los sesgos de selección.

En relación a las ayudas públicas a la innovación los resultados señalan que las cuatro alternativas consideradas (autonómica, estatal, europea y cualquier combinación de éstas) exhiben una influencia sobre la probabilidad de cooperar estadísticamente significativa en relación a la ausencia de financiación pública. Se verifica, asimismo, que dicho impacto es menor para las agencias auto- nómicas. Una posible explicación puede proceder de la existencia de múltiples agencias autonómicas en el ámbito territorial español, con posibles objetivos distintos, e incluso contradictorios, respecto del rol que deben desempeñar los acuerdos de cooperación en la difusión de la tecnología y en su contribución a la dinamicidad y competitividad de las correspondientes empresas. Sin embargo, la agencia estatal y la agencia europea, al mantener una identidad única, es factible que también tengan definidos unos objetivos claros y uniformes.

Los coeficientes obtenidos indican que el impacto sobre la probabilidad de cooperar de la agencia europea casi es el doble de la correspondiente agencia estatal española. Todo parece indicar, pues, que la agencia europea apoya más

\section{Tabla 2}

Resultados de la regresión del Modelo de interés

\begin{tabular}{|c|c|c|c|}
\hline & $\beta$ & Error estándar & Sig. \\
\hline Apoyo público & & & 0,000 \\
\hline Autonómico & 1,167 & 0,108 & 0,000 \\
\hline Estatal & 1,223 & 0,107 & 0,000 \\
\hline Europeo & 2,113 & 0,247 & 0,000 \\
\hline Combinado & 1,523 & 0,121 & 0,000 \\
\hline Spillovers entrantes & 0,290 & 0,044 & 0,000 \\
\hline Intensidad en I+D & 1,091 & 0,412 & 0,008 \\
\hline Costes y riesgos & 0,007 & 0,053 & 0,888 \\
\hline Complementariedades & $-0,077$ & 0,048 & 0,108 \\
\hline Métodos de protección legal & 0,122 & 0,045 & 0,007 \\
\hline Grupo & 0,467 & 0,088 & 0,000 \\
\hline Tamaño & 0,276 & 0,065 & 0,000 \\
\hline Constante & $-4,761$ & 0,510 & 0,000 \\
\hline
\end{tabular}

Fuente: Elaboración Propia a partir de la Base de Datos PITEC (2010). 
decididamente los acuerdos de cooperación en I+D que las demás agencias. Asimismo, cumple destacar que la combinación de dos o más agencias también exhibe un notable impacto sobre la probabilidad de cooperar, de lo que parece colegirse ciertos indicios de complementariedad entre las diferentes agencias en lo que a la instrumentación de acuerdos de cooperación se refiere.

La importancia del impacto que las ayudas públicas tienen sobre la probabilidad de cooperar resulta lógica. Así, resulta obvio que las empresas innovadoras se mueven habitualmente en las fronteras del conocimiento tecnológico, lo que les obliga a buscar recursos tecnológicos más allá de sus propios límites, pues es imposible generar internamente todos los recursos y capacidades necesarias para competir con éxito. Pero llevar a cabo exploraciones en las fronteras tecnológicas es costoso y arriesgado, y genera importantes externalidades y efectos de apropiabilidad indeseables (Klette et al., 2000), máxime cuando dicha exploración se realiza con la participación de socios externos. Precisamente, para paliar los efectos adversos que generan la apropiabilidad y las externalidades, resulta al efecto frecuente instrumentar ayudas públicas a la innovación, máxime cuando existe la anuencia generalizada de que la innovación constituye una de las claves de bóveda de la competitividad de las empresas, del crecimiento económico de los países y del bienestar de sus correspondientes habitantes (Heijs, 2005).

Por ello, muchas empresas que buscan la innovación a través de la instrumentación de acuerdos de cooperación reciben diversas clases de ayudas públicas. En concreto, en el ámbito español, la participación de fondos públicos en el desarrollo de actividades de I+D a nivel privado alcanza la cuota del 40\% (OCDE, 2001; Santamaría et al., 2010).

Los resultados obtenidos también permiten afirmar que los spillovers entrantes influyen positiva y significativamente sobre la probabilidad de cooperar en I+D de las empresas, lo que corrobora que la internalización de los flujos entrantes de información y conocimiento constituye uno de los objetivos clave que persiguen las empresas a la hora de establecer acuerdos de cooperación en I+D.

La hipótesis de que la existencia de una mayor intensidad tecnológica facilita que las empresas sean más propensas a establecer acuerdos de cooperación en I+D es confirmada por la significación estadística del correspondiente coeficiente, el más influyente sobre la probabilidad de cooperar después de las ayudas públicas a la innovación. Bajo la asunción de que dicha intensidad constituye una variable que mide la capacidad de absorción, este estudio confirma la importancia que la disponibilidad de un fuerte departamento interno de I+D tiene sobre la capacidad de las empresas para aprovechar, mediante el establecimiento de acuerdos de cooperación, el conocimiento que se genera extramuros de las empresas.

Este hallazgo resulta coherente con lo que la literatura económica sobre innovación al respecto preconiza, al advertir que la mayoría de los acuerdos de cooperación en I+D tienen lugar en los sectores de mayor complejidad tecnológica (Hagedoorn, 2002; Moskalev y Swensen, 2006). 
Sin embargo, el resultado que el estudio arroja sobre los obstáculos a la innovación, evaluados mediante la variable costes y riesgos, no es estadísticamente significativo, no cumpliéndose al respecto la hipótesis planteada. En el análisis que realizó López (2008) para la industria manufacturera española, la variable costes y riesgos era positiva y estadísticamente significativa. La discrepancia de resultados entre ambos estudios puede tener su origen en el tiempo histórico en el que está centrado cada análisis (años 2000 y 2010 , respectivamente) y en la diferente estrategia econométrica empleada. En López (2008) se llevó a cabo una estimación en dos etapas con utilización de variables instrumentales, a fin de tratar la problemática de potenciales variables endógenas.

En este estudio, se lleva a cabo una estimación en dos etapas con la finalidad de corregir los potenciales sesgos de selección. Por consiguiente, en el presente estudio se recoge la posible disímil percepción que sobre los obstáculos a la innovación tienen las empresas no innovadoras y, por tanto, su probable impacto sobre los acuerdos de cooperación en I+D. En el estudio de López (2008), únicamente se refleja la percepción sobre los obstáculos a la innovación de las empresas innovadoras.

En relación a la variable complementariedades, evaluada como un obstáculo a la innovación derivado de la escasez/disponibilidad de información tecnológica a nivel interno de cada empresa, sucede otro tanto de lo mismo, ya que dicha variable no es estadísticamente significativa. Por consiguiente, las hipótesis planteadas sobre costes y riesgos y com- plementariedades no encuentran refrendo estadístico en este estudio. Las discrepancias encontradas entre ambos estudios respecto del impacto de los obstáculos a la innovación sobre la probabilidad de cooperar en I+D sugieren que las motivaciones para cooperar de las empresas innovadoras pueden ser distintas de las empresas que no innovan.

Con respecto a los métodos de protección legal los resultados obtenidos indican que su influencia sobre la probabilidad de cooperar en I+D es positiva y estadísticamente significativa.

Asimismo, se colige de los resultados que las organizaciones empresariales que pertenecen a grupos de empresas tienen una mayor probabilidad de establecer acuerdos de cooperación que aquellas otras organizaciones que tienen un desempeño independiente. De hecho, esta variable es la tercera en importancia a la hora de explicar la propensión a cooperar en I+D, solo por detrás de las ayudas públicas a la innovación y de la corespondiente intensidad en I+D.

Esta influencia positiva y significativa confirma nuestra hipótesis de partida y, en ciertos aspectos, resulta lógica, en la medida en que las organizaciones que pertenecen a grupos de empresas tienen mayores facilidades para captar y ofrecer confianza a potenciales socios, así como acceso a informaciones internas del grupo que le capacitan para mantener negociaciones complejas e incrementar su potencial innovador (Piga y Vivarelli, 2004).

Finalmente, la influencia del tamaño sobre la probabilidad de cooperar también es positiva y estadísticamente signi- 
ficativa, al igual que ocurre en el estudio de López (2008). Por tanto, cabe resaltar que, en el contexto del sector manufacturero español, las empresas de mayor tamaño cooperan más en I+D que las empresas de menor tamaño.

\section{Conclusiones}

En este trabajo se analizan los determinantes más relevantes de los acuerdos de cooperación en I+D, utilizando para tal menester una muestra de empresas españolas manufactureras innovadoras procedentes de los microdatos del Panel de Innovación Tecnológica (PITEC) del año 2010. Además de explorar las variables más usuales en el ámbito de la cooperación en I+D, en el estudio empírico se ha analizado la influencia de las ayudas públicas a la innovación, una variable poco frecuente en esta clase de trabajos académicos, a pesar de la enorme importancia que las diferentes intervenciones públicas han adquirido en el ámbito de la promoción de las actividades de innovación.

En el estudio se han contrastado un total de seis hipótesis, habiéndose encontrado confirmación positiva sobre cuatro de estas hipótesis. Se ha analizado la significatividad estadística de dos variables (métodos de protección legal y tamaño) sobre las que la literatura existente no permite aventurar el signo de su influencia sobre la cooperación en I+D. Sobre el particular, se ha verificado que esta influencia es positiva y significativa. También se ha confirmado la influencia de los spillovers sobre la probabilidad de cooperar en I+D, de modo que su existencia empuja a las empresas al estableci- miento de esta clase de acuerdos al objeto de internalizar las externalidades que estos spillovers generan.

El análisis empírico se ha llevado a cabo mediante la realización de una regresión logística binaria de naturaleza bietápica, con la finalidad de eliminar el potencial sesgo de selección que pudiera estar presente, ya que la submuestra que se maneja en el estudio empírico sólo está compuesta de empresas innovadoras, en la medida en que son esta clase empresas las que exhiben una mayor propensión a cooperar en I+D.

De los resultados obtenidos se infiere que las empresas que exhiben una mayor probabilidad de cooperar en I+D son aquellas que reciben alguna clase de ayuda pública a la innovación, realizan mayores gastos internos en investigación y desarrollo, pertenecen a un grupo de empresas, tienen un mayor tamaño y utilizan métodos de protección legal para evitar que otras empresas se apropien de los resultados de sus investigaciones.

Cabe reseñar que de la consideración conjunta de los resultados obtenidos en este estudio y del reconocimiento de que la industria manufacturera española está conformada por la presencia mayoritaria de empresas de reducida dimensión, generalmente pertenecientes a sectores de media o baja intensidad tecnológica, es posible extraer algunas líneas de acción que pueden servir de guía para la instrumentación de medidas de política económica tendentes a promover la innovación de las empresas españolas y el establecimiento de acuerdos de cooperación en I+D entre las mismas. Destacar, al respecto, dos de estas posibles líneas de acción: 
1. Si se desea promover la existencia de empresas innovadoras y crear las condiciones para que el conocimiento de estas empresas se derrame o irradie sobre otras muchas empresas del tejido productivo, la instrumentación de políticas de ayudas públicas a la innovación, condicionadas al establecimiento de acuerdos de cooperación, aparentemente parece una medida correcta. Sobre el particular, los resultados del estudio realizado nos señalan que de todas las variables analizadas las ayudas públicas son las que tienen una mayor influencia sobre la probabilidad de que las empresas cooperen.

Sin embargo, es posible que en España muchas empresas pequeñas y de baja intensidad tecnológica se asocien porque la cooperación constituye una condición ineludible que deben cumplir para poder captar fondos públicos. Pero dado su exiguo acervo tecnológico, poca difusión de la tecnología puede esperarse de los acuerdos en I+D que se establecen entre estas clase de empresas. Por ejemplo, de los 1421 acuerdos de cooperación en I+D contabilizados en este estudio tan sólo 170 han estado protagonizados por las empresas de alta intensidad tecnológica.

2. La literatura sobre innovación suele resaltar que la capacidad de innovar de las empresas depende de forma crucial de la realización de fuertes inversiones en la creación de un departamento interno de I+D, del cual a su vez depende la capacidad de la empresa para reconocer y explotar el conocimiento valioso que potenciales socios puedan aportar. Sin embargo, como se ha detectado en este estudio, la intensidad en I+D media de las empresas tecnológicas españolas que han establecido acuerdos de cooperación tan solo es de 0,0496 sobre un valor máximo posible de 3,52 .

Por consiguiente, resulta razonable pensar que la instrumentación de políticas económicas tendentes a forzar la cooperación indiscriminada de empresas para tratar de conseguir una mayor difusión de la tecnología no puede dar los frutos apetecidos en el contexto del sector manufacturero español.

La secuencia lógica de esta clase de políticas parece otra: primero, ayudar a las pequeñas empresas a construir y fortalecer un departamento potente de $I+D$, otorgando las ayudas públicas a la innovación sin la condicionalidad de establecer acuerdos de cooperación; segundo, cuando estas empresas hayan desarrollado una capacidad de innovación aceptable, seguir concediéndoles ayudas públicas a la innovación, aunque ahora sí bajo la condicionalidad del establecimiento de acuerdos de cooperación en I+D; tercero, en los sectores de alta intensidad tecnológica la concesión de ayudas públicas a la innovación puede, y posiblemente debe, llevar aparejada la condicionalidad de establecer acuerdos de cooperación.

Finalmente, resaltar que además de una utilización inteligente y selectiva de la concesión de ayudas públicas a la innovación bajo la condicionalidad de la cooperación, las políticas públicas tendentes a coadyuvar a la difusión de la tec- 
nología también pueden dirigirse al establecimiento de ayudas y condiciones que propicien el crecimiento de las empresas, principalmente desde coordenadas financieras, pues el tamaño de las empresas tiene una notable influencia sobre la probabilidad de cooperar en I+D.

\section{Referencias Bibliográficas}

Abramovsky, Laura; Harrinson, Rupert y Simpson, Helen (2004). "Increasing innovative activity in the Uk? Where now for government support for innovation and technology transfer?", Institute for Fiscal Studies, Briefing Note, $\mathrm{n}^{\circ}$ 53, London, UK., November.

Acosta, Juan y Modrego, Aurelia (2001). "Public financing of cooperative R\&D projects in Spain: the concerted projects under the national R\&D plan", Research Policy, vol. $30, \mathrm{n}^{\circ} 4$, pp. 625-641.

Anbarci, Nejat; Lemke, Robert y Roy, Santanu (2002). "Inter-firm complementarities in R\&D: A re-examination of the relative performance of joint ventures", International Journal of Industrial Organization, vol. 20, n² 2, pp. 191-213.

Arora, Ashish y Gambardella, Alfonso (1994). "Evaluating technological information and utilizing it: Scientific knowledge, technological capability and external linkages in biotechnology", Journal of Economic Behavior and Organization, vol. 24, n 1, pp. 91-114.

Arrow, Kenneth (1959), Economic Welfare and the allocation of resources for invention, Mimeo, Rand Corporation.

Bayona, Cristina; García, Teresa y Huerta, Emilio (2002). "Collaboration in R\&D with universities and research centres: an empirical study of Spanish firms", R\&D Management, vol. 32, pp. 321-341.
Bayona, Cristina; Corredor, Pilar y Santamaría, Rafael (2006). "Technological alliances and the market valuation of new economy firms", Technovation, vol. 26, n 3, pp. 369-383.

Belderbos, Rene; Carree, Martin; Diederen, Bert; Lokshin, Boris y Veugelers, Reinhilde (2004). "Heterogeneity in R\&D cooperation strategies", International Journal of Industrial Organization, vol. 22, n. 8/9, pp. 1237-1263.

Bönte, Werner y Keilbach, Max (2005). "Concubinage or marriage? Informal and formal cooperation for innovation", International Journal of Industrial Organization, vol. 23, n³/4, pp. 279-302.

Caloghirou, Yannis; Kastelli, loanna y Tsakanikas, Aggelos (2004). "Internal capability and external knowledge sources: complements or substitutes for innovative performance?" Technovation, vol. 24, no 1, pp. 29-39.

Cassiman, Bruno y Veugelers, Reinhilde (2002). "R\&D cooperation and spillovers: some empirical evidence from Belgium", American Economic Review, vol. 92, n 4, pp. 1169-1184.

Cassiman, Bruno y Veugelers, Reinhilde (2006). "In search of complementarity in innovation strategy: internal R\&D and external knowledge acquisition", Management Science, vol. 52, pp. 68-82.

Cohen, Wesley y Levinthal, Daniel (1989). "Innovation and learning: the two faces of R\&D", Economic Journal, vol. 99, $\mathrm{n}^{\circ}$ 397, pp. 569-596.

Cohen, Wesley y Levinthal, Daniel (1990), “Absorptive capacity: a new perspective on learning and innovation", Administrative Science Quarterly, vol. 35, $\mathrm{n}^{\circ}$ 1, pp. 128-152.

Colombo, Massimo y Garrone, Paola (1998). "A simultaneous equation model of technological agreements and infra-mural R\&D", en Colombo, M. (Ed.): The 
changing boundaries of the firm, Routledge, London, pp. 140-157.

Cowan, Robin y Jonard, Nicolas (2008). "If the Alliance Fits. Innovation and Network Dynamics", UNU-Merit, Working Paper Series, No. 2008-022, Maastricht, The Netherlands.

Dachs, Bernhard; Ebersberger, Bernd y Pyka, Andreas (2008). "Why do firms cooperate for innovation?-a comparison of Austrian and Finnish CIS3 results", International Journal of Foresight and Innovation Policy, vol. 4, $n^{\circ} 3 / 4$, pp. 200-229.

Das, Tushar Kanti y Teng, Bing-Sheng (2000). "A resource-based theory of strategic alliances", Journal of Management, vol. 26, $n^{\circ}$ 1, pp. 31-61.

D'Aspremont, Claude y Jacquemine, Alexis (1988). "Cooperative and noncooperative $R \& D$ in duopoly with spillovers", The American Economic Review, vol. $78, n^{\circ} 5$, pp. 1133-1137.

Falk, Martin (2004). "What drives business R\&D intensity across OCDE countries?" Applied Economics, vol. 35, n 5, pp. 533-577.

Faria, Pedro; Lima, Francisco y Santos, Rui (2010). "Cooperation in innovation activities: The importance of partners", Research Policy, vol. 39, $\mathrm{n}^{\circ} 8$, pp. 1082-1092.

Fritsch, Michael y Lukas, Rolf (2001). "Who cooperates on R\&D?" Research Policy, vol. 30, n² 2, pp. 297-312.

Gemünden, Hans Georg; Heydebreck, Peter y Herden, Rainer (1992). "Technological interweavement: a means of achieving innovation success", R\&D Management, vol. 22, $n^{\circ} 4$, pp. 359-375.

Georghiou, Luke y Roessner, David (2000). "Evaluating technology programs: tools and methods", Research Policy, vol. 29, $n^{\circ} 4-5$, pp. 657-678.

Greene, William (1993). Econometric Analysis, New York: Macmillan.
Hagedoorn, John (1993). "Understanding the rationale of strategic technology partnering: interorganizational modes of cooperation and sectoral differences", Strategic Management Journal, vol. 14, no. 5, pp. 371-385.

Hagedoorn, John (2002). "Inter-firm R\&D partnerships: an overview of major trends and patterns since 1960", Research Policy, vol. 31, n 4 , pp. 477-492.

Haas, Martine y Hansen, Morten, (2005). "When using knowledge can hurt performance: The value of organizational capabilities in a management consulting company", Strategic Management Journal, vol. 26, pp. 1-24.

Heijs, Joost (2005). "Do public policies that foster co-operation in innovation augment the co-operative attitude: The empirical facts", Documentos de trabajo del Instituto de Análisis Industrial y Financiero, n?, 52, Madrid.

Henderson, Rebecca y Cockburn, lain (1996). "Scale, Scope, and Spillovers: The Determinants of Research Productivity in Drug Discovery", The Rand Journal of Economics, vol. 27, $n^{\circ} 1$, pp. 32-59.

Hite, Julie y Hesterly, William (2001). "The evolution of firm networks: From emergence to early growth of the firm", Strategic Management Journal, vol. 22, $n^{\circ} 3$, pp. 275-286.

Kim, Linsu (2001). "The dynamics of technological learning in industrialization”, International Social Science Journal, vol. 53, n 168, pp. 297-308.

Kleinknecht, Alfred y Reijnen, Jeroen (1992). "Why do firms cooperate on R\&D: an empirical study", Research Policy, vol. 21, no 4, pp. 347-360.

Klette, Tor Jakob; Moen, Jarle y Griliches, Zvi (2000). "Do subsidies to commercial $\mathrm{R} \& D$ reduce market failures? Microeconomic evaluation studies", Research Policy, vol. 29, n 4/5, pp. 471-495. 
López, Alberto (2008). "Determinants of R\&D cooperation: evidence from Spanish manufacturing firms", International Journal of Industrial Organization, vol. 26, n 1, pp. 113-136.

Luukkonen, Terttu (2000). "Additionality of EU framework programmes", Research Policy, vol. 29, n 6, pp. 711-724.

Manning, Willard; Duan, Naihu y Rogers, William, (1987). "Monte Carlo evidence on the choice between sample selection and two-part models", Journal of Econometrics, vol. 35, pp. 59-82.

Miotti, Luis y Sachwald, Frederique (2003). "Co-operative R\&D: why and with whom? An integrated framework of analysis", Research Policy, vol. 32, $n^{\circ} .8$, pp. 1481-1499.

Mohnen, Pierre y Hoareau, Cathy (2003). "What type of enterprise forges close links with universities and government labs? Evidence from CIS 2", Managerial and Decision Economics, vol. 24, n² 2-3, pp. 133-145.

Moskalev, Sviatoslav y Swensen, Bruce (2006). "Joint ventures around the globe from 1990-2000: Forms, types, industries, countries and ownership patterns", Review of Financial Economics, vol. 16, n 1, pp. 29-67.

Negassi, Syoum (2004). "R\&D co-operation and innovation: a microeconometric study on French firms", Research Policy, vol. 33, n³, pp. 365-384.

Organization for Economic Cooperation and Development (OECD) and Eurostat (1997). Oslo Manual: Proposed Guidelines for Collecting and Interpreting Technological Innovation data, Paris.

Organization for Economic Cooperation and Development (OECD) (2001). OECD Science, technology and industry scoreboard. Towards a knowledge based economy, Paris.
Okamuro, Hiroyuki; Kato, Masatoshi y Honjo, Yuji (2011). "Determinants of R\&D cooperation in Japanese start-ups", Research Policy, vol. 40, n 5, pp. 728-738.

Panel de Innovación Tecnológica (PITEC) (2010). Fundación para la Ciencia y la Tecnología (FECYT), Madrid.

Piga, Claudio y Vivarelli, Marco (2004). "Internal and External R\&D: A Sample Selection Approach", Oxford Bulletin of Economics and Statistics, vol.66, $\mathrm{n}^{\circ}$ 4, pp. 457-482.

Reger, Guido y Kuhlmann, Stefan (1995). "European technology policy in Germany: the impact of European Community policies upon science and technology in Germany", Physica-Verlag GmbH \& Co., Berlin, Germany.

Sampson, Rachelle (2007). "R\&D alliances and firm performance: the impact of technological diversity and alliance organization on innovation", Academic of Management Journal, vol. 50, $\mathrm{n}^{\circ}$ 2, pp. 364-386.

Santamaría, Lluis; Barge-Gil, Andrés y Modrego, Aurelia (2010). "Public selection and financing of R\&D cooperative projects: Credit versus subsidy funding", Research Policy, vol. 39, $\mathrm{n}^{\circ} 4$, pp. 549-563.

Schoenmakers, Wilfred y Duysters, Geert (2006). "Learning in strategic technology alliances", Technology Analysis \& Strategic Management, vol. $18, n^{\circ} 2$, pp. 245-264.

Stock, Gregory; Greis, Noel y Fisher, William (2001). "Absorptive capacity and new product development", Journal of High Technology Management Research, vol. 12, $\mathrm{n}^{\circ} 1$, pp. 77-91.

Tether, Bruce (2002). "Who co-operates for innovation, and why. An empirical analysis", Research Policy, vol. 31, $n^{\circ}$ 6, pp. 947-967. 
Ayudas públicas como determinante de la cooperación en investigación...

Guisado G., Manuel; Guisado T., Manuel y Ferro S., Carlos

Tsai, Kuen-Hung (2009). "Collaborative networks and product innovation performance: toward a contingency perspective", Research Policy, vol. $38, \mathrm{n}^{\circ}$ 5, pp. $765-778$.
Yi, Sang-Seung y Shin, Hyukseung (2000). "Endogenous formation of research coalitions with spillovers", International Journal of Industrial Organization, vol. $18, n^{\circ} 2$, pp. 229-256. 